\title{
Simulator-based training for learning resuscitative transesophageal echocardiography
}

\author{
Jordan Chenkin, MD, MEd†; Edgar Hockmann, MD*; Tomislav Jelic, MD ${ }^{\S}$
}

\section{CLINICIAN'S CAPSULE}

What is known about the topic?

Transesophageal echocardiography (TEE) is a relatively new tool for emergency physicians that may assist with resuscitations.

What did this study ask?

Is a 1-hour simulation-based teaching session effective for teaching resuscitative TEE to emergency medicine residents?

What did this study find?

Emergency medicine residents had a high success rate for generation of TEE images on a simulator after a brief training session.

Why does this study matter to clinicians?

This study demonstrates that TEE may be relatively easy to learn for emergency medicine residents who are already proficient with ultrasound.

\section{ABSTRACT}

Objectives: Transesophageal echocardiography (TEE) is a relatively new resuscitation tool in the emergency department. Recent studies have demonstrated that it can impact diagnosis and management of critically ill patients. The objective of this study is to determine the effectiveness of a simulation-based curriculum for teaching emergency medicine residents a five-view TEE protocol.

Methods: Emergency medicine residents with previous ultrasound experience were invited to attend a 1-hour TEE training session. The training consisted of a didactic lecture followed by guided practice on a simulator. Performance was measured prior to training, after the training session, and by a transfer test 1 to 2 weeks after training. The primary outcome was the percentage of successful image generation using a scoring tool by two blinded reviewers.

Results: Twenty-two residents completed the study. The percentage of successful views increased from $44.5 \%$ (SD 27.9) at baseline to $98.6 \%$ (SD 3.5$)$ after training $(p<0.001)$, and was $86.8 \%$ (SD 12.1) on transfer testing ( $p<0.001)$.

Conclusion: A brief simulation-based teaching session was effective for teaching emergency medicine residents a fiveview resuscitative TEE protocol. Future studies are needed to determine optimal methods for long-term skill retention.

\section{RÉSUMÉ}

Objectif: Le recours à l'échocardiographie transœsophagienne (ETO) dans les cas de réanimation est relativement nouveau au service des urgences. D'après des études récentes, les résultats de l'examen peuvent avoir une incidence sur le diagnostic et la prise en charge de maladies très graves. L'étude visait donc à déterminer l'efficacité d'une séance de formation par simulation sur un protocole d'ETO en cinq prises de vue à des résidents en médecine d'urgence (MU).

Méthode: Des résidents en MU ayant une expérience antérieure en échographie ont été invités à participer à une séance d'une heure sur l'ETO. Celle-ci se divisait en deux parties : enseignement magistral et application pratique guidée sur simulateur. Une mesure de la performance a été effectuée avant et après la séance de formation, puis de 1 à 2 semaines après celle-ci à l'aide d'un test d'application des connaissances. Le critère principal consistait dans le pourcentage de la production d'images réussies d'images et en leur évaluation par deux examinateurs tenus dans l'ignorance des faits, à l'aide d'une méthode de pointage.

Résultats: Au total, 22 résidents ont terminé l'étude. Le pourcentage de prises de vue réussies est passé de $44,5 \%$ (écarttype $[\sigma]=27,9)$ au départ à $98,6 \%(\sigma=3,5)$ après la formation $(p<0,001)$, et celui-ci a atteint $86,8 \%(\sigma=12,1)$ au test d'application des connaissances $(p<0,001)$.

Conclusion: La tenue d'une brève séance de formation par simulation s'est révélée efficace dans l'enseignement d'un protocole d'ETO en cinq prises de vue à des résidents en MU. II faudrait toutefois mener d'autres études afin de déterminer les meilleures méthodes d'enseignement possible favorisant la mémorisation de la matière à long terme.

From the *Sunnybrook Health Sciences Centre, Toronto, ON; †Division of Emergency Medicine, University of Toronto; $¥$ Department of Anaesthesia, University of Toronto, Toronto, ON; and the §Department of Emergency Medicine, University of Manitoba, Winnipeg, MB.

Correspondence to: Dr. Jordan Chenkin, Sunnybrook Health Sciences Centre, University of Toronto, 2075 Bayview Avenue, AG245, Toronto, ON M4N 3M5; Email: Jordan.chenkin@utoronto.ca

(C) Canadian Association of Emergency Physicians 
Keywords: Education, simulation, transesophageal

echocardiography

\section{INTRODUCTION}

Point-of-care ultrasound (POCUS) is increasingly being used to guide the management of critically ill patients in the emergency department (ED). A rapid evaluation of the cardiovascular structures with POCUS can help emergency physicians (EPs) rapidly rule in and rule out life-threatening causes of shock. The use of resuscitative POCUS is becoming a critical skill for EPs and is a recommended core competency for residency training programs. ${ }^{1}$

Recently, transesophageal echocardiography (TEE) has been studied as a potentially useful adjunct to resuscitative POCUS. ${ }^{2}$ Potential advantages of TEE include more reliable image generation, higher resolution images, and the ability to continuously monitor cardiac function, even during chest compressions. ${ }^{3}$

Prior studies have demonstrated that simulator-based training is an effective method for learning focused TEE amongst practicing EPs. ${ }^{4}$ As more EDs begin to adopt the use of TEE, there is also a need to develop education protocols for emergency medicine trainees. Currently, there are limited data on TEE training programs for emergency medicine residents. The objective of this study is to determine the effectiveness of a simulator-based curriculum for teaching residents a focused resuscitative TEE protocol.

\section{METHODS}

\section{Study design and setting}

We conducted a prospective observational cohort study. All emergency medicine residents rotating through a single academic health sciences centre were invited to participate. Inclusion criteria included having completed their core emergency ultrasound rotation and an elective in advanced ultrasound, which includes a requirement of performing a minimum of 25 transthoracic echocardiograms. Participants were excluded if they had previous training or exposure to TEE. The study was approved by the hospital research ethics board.

\section{Intervention}

Participants completed a baseline questionnaire followed by an assessment of their TEE skills using a commercially available simulator (Vimedix, CAE Healthcare, Ville St-Laurent, QC). They individually attended a 1-hour training session on a resuscitative TEE protocol, consisting of a didactic lecture by an EP with extensive TEE experience, followed by guided practice using the simulator. The TEE scanning protocol included a five-view sequence, including the midesophageal four-chamber, mid-esophageal long axis, transgastric short axis, bicaval, and thoracic aorta views. This was similar to a previously published four-view exam, with the addition of the thoracic aorta view. ${ }^{4}$ Following the training, they completed an assessment of their skills by performing a sequence of views without feedback. To assess knowledge transfer, participants returned for another assessment at 1 to 2 weeks after the initial training. In this assessment, they were provided with two patient scenarios (cardiogenic shock and massive pulmonary embolism) and asked to determine the diagnosis using the resuscitative TEE protocol. All assessments were video recorded for a subsequent blinded review.

\section{Outcome measures and analysis}

The primary outcome for this study was the percentage of successful views, as determined by two blinded reviewers using an anchored scoring tool. The reviewers included a cardiac anesthesiologist board-certified in TEE and an EP with significant TEE training and experience. The scoring tool was modified from a previously validated ultrasound assessment tool. ${ }^{5}$ A successful view was defined as being of sufficient quality to allow for a clinical interpretation. Secondary outcomes included a correct diagnosis on transfer testing, time to complete the scan, and success rates for individual views. The chi-square test and student's t-test were used for categorical and continuous data with a statistical significance set at 0.05 . 


\section{RESULTS}

A total of 25 residents were invited to participate, and 22 (88\%) completed the study. Two residents declined to participate, and one was ineligible due to previous TEE experience. Years of training ranged from PGY-3 to PGY-5 (mean 4.5), and 21 (95\%) had an independent practitioner certification for POCUS. The median time between training and transfer test was 9.5 days (interquartile range of 7-12).

The percentage of successful views increased from $44.5 \%$ (SD 27.9) at baseline to $98.6 \%$ (SD 3.5) after training $(p<0.001)$ and was $86.8 \%$ (SD 12.1) on transfer testing $(p<0.001)$. The time to complete the scan was 330 seconds at baseline, 125 seconds after training $(p<$ $0.001)$, and 184 seconds $(p<0.001)$ in the transfer test. Participants made the correct diagnosis in $75 \%$ (SD 25.6) of the cases in the simulated patient encounter in the transfer test. The descending aorta view had the highest success rate (93.2\%), and the mid-esophageal long axis view had the lowest success rate $(75.0 \%)$ (Figure 1).

\section{DISCUSSION}

In this study, we found that after a 1-hour teaching session, emergency medicine residents were successful at learning a five-view resuscitative TEE protocol using a simulator. Currently, there is little evidence on optimal training methods for goal-directed TEE by emergency medicine residents. A policy statement published by the American College of Emergency Physicians (ACEP) suggested that training includes a minimum of 2 to 4 hours of didactic training, 10 proctored examinations on live patients, and an assessment by a credentialed provider. ${ }^{6}$ Despite these recommendations, it may be difficult for EPs and trainees to find the opportunity to perform training examinations on live patients.

When access to supervised training on live patients is limited, simulation-based education may be an effective complementary method of learning this technique. ${ }^{7-9}$ Matyal et al. used a high-fidelity simulator to train 18 anesthesiology residents with no prior TEE experience and subsequently assessed their TEE performance on live patients in the operating room. All participants

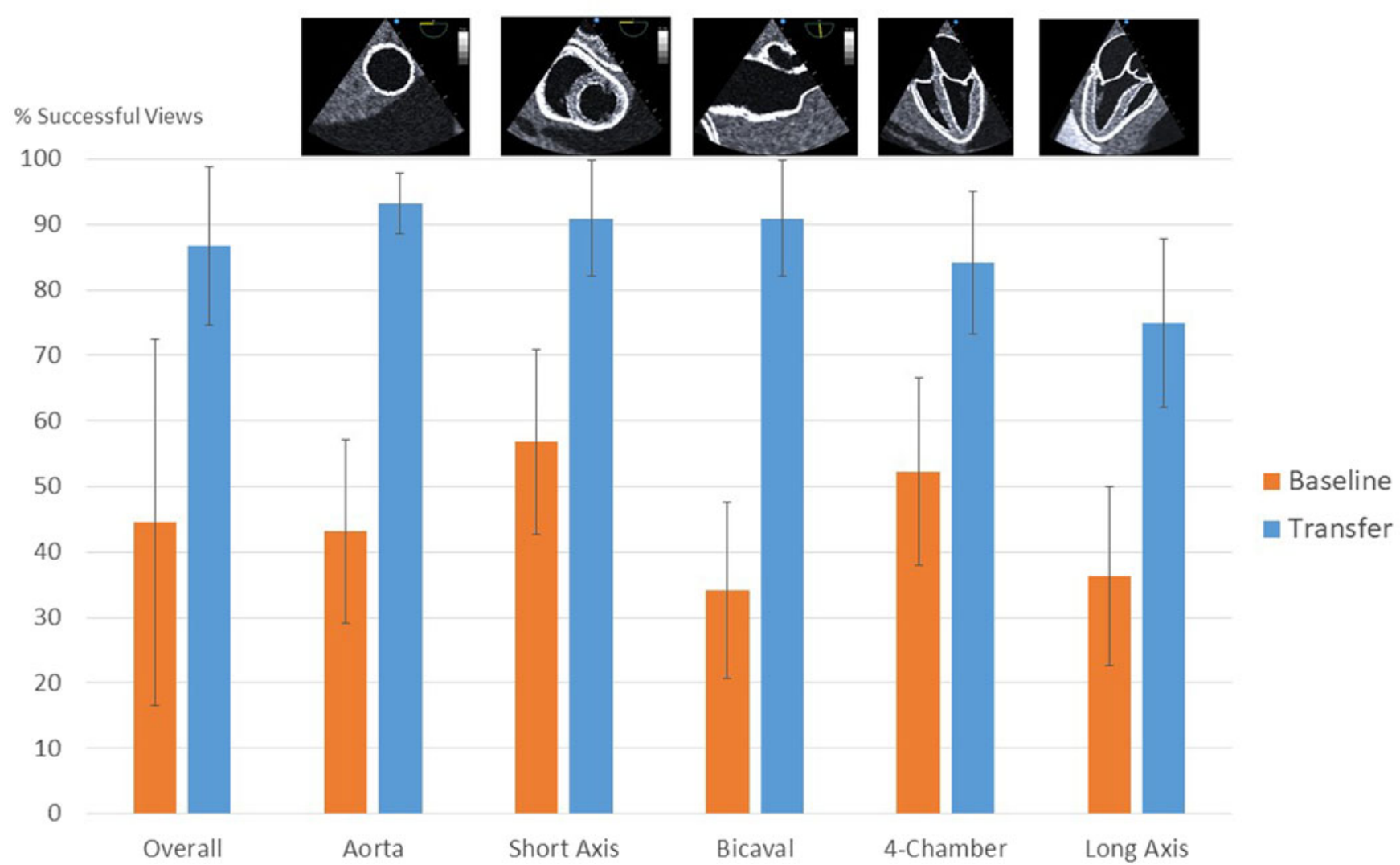

Figure 1. Success rates of individual TEE views at baseline and on transfer test. Thumbnail images representing each of the views are shown above the graph. 
were able to generate adequate TEE views without assistance as determined by review by blinded experts. ${ }^{10}$ In an educational study of $14 \mathrm{EPs}$, Arntfield et al. demonstrated that a 4-hour workshop was effective at teaching a four-view TEE scanning protocol. ${ }^{4}$ The same group then conducted a retrospective review of the use of TEE in the ED. They found that, despite having no live patient training, 12 EPs trained on using the simulator were able to generate determinate scans $98 \%$ of the time, and the scans facilitated a diagnosis in $78 \%$ of cases and impacted management in $67 \%$ of cases. $^{2}$

Unlike the study by Arntfield, we chose to include the thoracic aorta view in our scanning protocol. This view allows for a more detailed assessment of the aorta for aneurysms and dissections, and is relatively easy because it does not require any flexion or omniplane adjustments. We found that the mid-esophageal long axis view had a lower success rate than the other views. This view requires the operator to adjust the omniplane of the transducer, which is a transducer manipulation that is unique to TEE. Given the slightly lower success rates, future studies should evaluate whether a simpler view, such as the mid-esophageal five-chamber view, could be an effective alternative for an evaluation of the proximal aorta and aortic valve for major pathology.

While the participants demonstrated a very high success rate for image generation, we noted a lower rate for diagnostic accuracy of $75 \%$ on the transfer test. We believe that this was due to the focus on image generation and a lack of exposure to pathology in the training sessions. In the transfer test, the participants likely continued to focus on generating high-quality images instead of seeking out pathology.

\section{Limitations}

This study was limited by the small sample size, and the results may not be generalizable to a larger population of learners. The training sessions were performed one-on-one with each learner, therefore the success rates may not be generalizable to larger group training sessions. Due to resident scheduling restrictions, our transfer test occurred at a median of 9.5 days after the initial training, and therefore we cannot comment on long-term skill retention. The study used a simulator, and therefore the results may not translate into success rates on actual ED patients.

\section{CONCLUSIONS}

Our data suggest that simulation can be used to train emergency medicine residents in resuscitative TEE. While rates of successful image generation were very high, the accuracy of image interpretation was only moderate. Future studies are needed to determine optimal teaching methods to improve real-world performance and facilitate long-term retention of this skill.

Acknowledgements: The authors thank Agnes Ryzynski from the Sunnybrook Simulation Centre and resident participants from the Division of Emergency Medicine, University of Toronto, for their contribution to this study.

Competing interests: None declared.

\section{REFERENCES}

1. Olszynski P, Kim D, Chenkin J, Rang L. The core emergency ultrasound curriculum project: a report from the Curriculum Working Group of the CAEP Emergency Ultrasound Committee. CFEM 2018;20:176-82.

2. Arntfield R, Pace J, Hewak M, Thompson D. Focused transesophageal echocardiography by emergency physicians is feasible and clinically influential: observational results from a novel ultrasound program. 7 Emerg Med 2016;50:286-94.

3. Blaivas M. Transesophageal echocardiography during cardiopulmonary arrest in the emergency department. Resuscitation 2008;78:135-40.

4. Arntfield R, Pace J, McLeod S, et al. Focused transesophageal echocardiography for emergency physicians-description and results from simulation training of a structured four-view examination. Crit Ultrasound 7 2015;7:27.

5. Ziesmann MT, ParkJ, Unger BJ, et al. Validation of the quality of ultrasound imaging and competence (QUICk) score as an objective assessment tool for the FAST examination. $\mathcal{F}$ Trauma Acute Care Surg 2015;78:1008-13.

6. Fair J, Mallin M, Mallemat H, et al. Transesophageal echocardiography: guidelines for point-of-care applications in cardiac arrest resuscitation. Ann Emerg Med 2018;71:201-7.

7. Sohmer B, Hudson C, Hudson J, et al. Transesophageal echocardiography simulation is an effective tool in teaching psychomotor skills to novice echocardiographers. Can $\mathfrak{F}$ Anaesth 2014;61:235-41.

8. Smelt J, Corredor C, Edsell M, et al. Simulation-based learning of transesophageal echocardiography in cardiothoracic surgical trainees: a prospective, randomized study. 7 Thorac Cardiovasc Surg 2015;150:22-5.

9. Bloch A, von Arx R, Etter R, et al. Impact of simulator-based training in focused transesophageal echocardiography: a randomized controlled trial. Anesth Analg 2017;125:1140-8.

10. Matyal R, Mitchell JD, Hess PE, et al. Simulator-based transesophageal echocardiographic training with motion analysis: a curriculum-based approach. Anesthesiology 2014;121:389-99. 